\title{
Small ring has big potential: insights into extrachromosomal DNA in cancer
}

\author{
Yihao Wang ${ }^{1,2}$, Rui Huang ${ }^{1,2}$, Guopei Zheng ${ }^{1,2}$ and Jianfeng Shen ${ }^{1,2^{*}}$ (i)
}

\begin{abstract}
Recent technical advances have led to the discovery of novel functions of extrachromosomal DNA (ecDNA) in multiple cancer types. Studies have revealed that cancer-associated ecDNA shows a unique circular shape and contains oncogenes that are more frequently amplified than that in linear chromatin DNA. Importantly, the ecDNA-mediated amplification of oncogenes was frequently found in most cancers but rare in normal tissues. Multiple reports have shown that ecDNA has a profound impact on oncogene activation, genomic instability, drug sensitivity, tumor heterogeneity and tumor immunology, therefore may offer the potential for cancer diagnosis and therapeutics. Nevertheless, the underlying mechanisms and future applications of ecDNA remain to be determined. In this review, we summarize the basic concepts, biological functions and molecular mechanisms of ecDNA. We also provide novel insights into the fundamental role of ecDNA in cancer.
\end{abstract}

Keywords: EcDNA, Oncogene amplification, Chromosomal rearrangement, Epigenetic modification, Tumor heterogeneity

\section{Introduction}

Extrachromosomal DNA (ecDNA) is a particular type of DNA molecule outside of the chromosome that is usually 1-3 Mb in length [1]. EcDNA does not contain centromeres or telomeres, but it has regulatory regions that control the expression of the encoded genes [2,3]. Studies have shown that ecDNA accounts for nearly $30 \%$ of all DNA particles outside of the chromosome [4, 5]. In addition, there are two major categories of extrachromosomal DNA particles that differ from ecDNA in sequence length: (1) extrachromosomal small circular DNA (eccDNA) and (2) ring- or neochromosome (Table 1) [6-9]. eccDNA is a double-stranded circular molecule less than $1 \mathrm{Mb}$ in length that consists of multiple copies of genome-originated repetitive non-coding DNA sequences and telomeric circles (e.g. small polydispersed circular DNA and microDNA) [10]. In contrast to

*Correspondence: jfshen@shsmu.edu.cn

${ }^{1}$ Department of Ophthalmology, Ninth People's Hospital, Shanghai JiaoTong University School of Medicine, Shanghai 200025, China

Full list of author information is available at the end of the article
ecDNA that is rarely seen in normal cells, eccDNA is present in both normal cells and cancer cells, and eccDNA may promote tumorigenesis through the selection of telomere extension and modulation of genomic instability $[10,11]$. In ring-chromosomes, the ends of the DNA sequence are fused together to form a ring shape [12]. Neochromosomes contain centromere and telomere sequences, with a typical sequence length of $30-600 \mathrm{Mb}$ $[7,8]$. Neochromosomes have been shown to contain high copy numbers of oncogenes and can be created through chromothripsis [9].

Recent findings have revealed the essential roles of ecDNA in cancer [1, 2, 12-14]. ecDNA is widely expressed in multiple types of cancers, including highly aggressive glioblastoma and sarcoma, but not in normal tissues $[2,15]$. The presence of ecDNA is also associated with the rapid amplification of oncogenes and elevated intra-tumoral heterogeneity [15]. Moreover, the lack of centromeres in ecDNA leads to the discordant inheritance of ecDNA elements during mitosis, contributing to the hyper-activity of oncogene expression. These features of ecDNA endow cancer cells with the ability of quick 
Table 1 Characteristics of ecDNA, eccDNA, neo and ring chromosome

\begin{tabular}{|c|c|c|c|c|c|c|}
\hline & Size & $\begin{array}{l}\text { Single/double } \\
\text { strand }\end{array}$ & Sequence feature & Definition & Origination & Refs. \\
\hline ecDNA & $\begin{array}{l}\text { 1-3 Mb, visible } \\
\text { under } \\
\text { microscope }\end{array}$ & Double & $\begin{array}{l}\text { Oncogene amplifi- } \\
\text { cation, regula- } \\
\text { tory regions, no } \\
\text { centromeres or } \\
\text { telomeres }\end{array}$ & $\begin{array}{l}\text { Extrachromosomal } \\
\text { DNA (double } \\
\text { minutes) }\end{array}$ & $\begin{array}{l}\text { BFB cycle, translo- } \\
\text { cation-deletion- } \\
\text { amplification, } \\
\text { episome and } \\
\text { chromothripsis }\end{array}$ & {$[1,3]$} \\
\hline eccDNA & $\begin{array}{l}<1 \mathrm{Mb} \text {. Invisible } \\
\text { under microscope }\end{array}$ & Single or double & $\begin{array}{l}\text { Small regulatory } \\
\text { RNA }\end{array}$ & $\begin{array}{l}\text { Extrachromosomal } \\
\text { small circular DNA }\end{array}$ & $\begin{array}{l}\text { Telomere circle, } \\
\text { spcDNA, miDNA, } \\
\text { episome }\end{array}$ & {$[93,94]$} \\
\hline $\begin{array}{l}\text { Neo } \\
\text { chromosome }\end{array}$ & $\begin{array}{l}\text { 30-600 Mb, visible } \\
\text { under microscope }\end{array}$ & Double & $\begin{array}{l}\text { Contains cen- } \\
\text { tromere or } \\
\text { telomere }\end{array}$ & $\begin{array}{l}\text { Structurally abnor- } \\
\text { mal chromosome }\end{array}$ & $\begin{array}{l}\text { Chromothripsis } \\
\text { and BFB cycles } \\
\text { with telomere } \\
\text { aggregation }\end{array}$ & {$[7,9]$} \\
\hline Ring chromosome & $\begin{array}{l}\text { 1.4-7.3 cms. Visible } \\
\text { under microscope }\end{array}$ & Double & $\begin{array}{l}\text { Circular or linear } \\
\text { form, contains } \\
\text { centromere and } \\
\text { telomere }\end{array}$ & $\begin{array}{l}\text { Breaks of telomeric } \\
\text { ends, end-to-end } \\
\text { fusion of the cen- } \\
\text { tric chromosome }\end{array}$ & $\begin{array}{l}\text { End joining of DNA } \\
\text { double-strand } \\
\text { breaks, telomere_- } \\
\text { subtelomere } \\
\text { junction, or rear- } \\
\text { rangement }\end{array}$ & [6] \\
\hline
\end{tabular}

adaptation toward the microenvironment, therefore promoting intra-tumoral heterogeneity $[1,16]$. The study of ecDNA in cancer is still in its infancy. In this review, we summarize the recent findings of ecDNA regarding the structure, biogenesis, function and therapeutic potentials in cancer.

\section{The biological features of ecDNA \\ Structure}

Early attempts to uncover the structure of ecDNA were limited due to technical obstacles. Recent advances in next-generation sequencing technologies (e.g. whole genome sequencing) and computational analytical approaches have led to the discovery that ecDNA presents in a circular shape and can replicate independently outside of chromosomes $[2,16,17]$. In addition, ecDNA contains not only complete genes, but also regulatory elements such as upstream promoters and enhancers $[2,3]$. Importantly, rewiring of adjacent enhancers along with endogenous enhancers was observed in ecDNA [3]. In certain circumstances, ecDNA can incorporate DNA segments from different chromosomes to form chimeric sequences, which may subsequently "invade" and re-integrate into other chromosomes to generate novel DNA sequences [2]. In comparison with linear DNA, ecDNA has a highly accessible chromatin state and significantly higher levels of H3K27ac, a well-established histone marker for super enhancers $[2,3]$. These structural characteristics of ecDNA markedly elevate the expression levels of oncogenes in ecDNA and affect chromatin rearrangement to promote intra-tumoral heterogeneity [16]. Verhaak et al. analyzed circular DNA data from 3,212 patients across a variety of cancer types. The association between oncogene amplification and ecDNA structure was observed, however such association could not be applied to the breakpoint; and the distributions of oncogene amplicons were highly nonrandom [15]. These results demonstrate that not only the inherited "genetic code," but also the topology and three-dimensional chromatin landscape play critical roles in maintaining proper function of the cancer genome $[1,15,18]$.

\section{Biogenesis}

The biological source of ecDNA generation includes endogenous DNA damage (e.g. DNA replication stress), exogenous stress (e.g. carcinogens and pathogens) and aberrations in the DNA damage repair machinery [19]. Both ecDNA and homogeneously staining regions (HSR) of chromosomes can be formed through gene transcription and dramatically increase the complexity and plasticity of the genome [20-22]. Nevertheless, the underlying mechanisms of ecDNA biogenesis are not fully understood. In addition to simple self-ligation after DNA breaks, ecDNA can also be generated in multiple other ways [23, 24], and several models have been proposed, such as the breakage-fusion-bridge cycle [25], translocation-deletion-amplification [26], episome [27] and chromothripsis [28] models. The diverse genome compositions of ecDNA in multiple cancers imply complex multiple-step procedures in its formation, including the generation of DNA fragments from DNA damage (e.g. double-strand breaks), tandem duplication [29], breakage-fusion-bridge cycle [30] and chromothripsis-mediated chromatin rearrangement 
[31]. Non-homologous end-joining or microhomologymediated end-joining repair mechanisms facilitate the rewiring of these DNA fragments in a random order, contributing to the generation of ecDNA [15, 32]. Importantly, ecDNA can self-replicate in the absence of tumor suppressors [1]. However, contradictory results have been reported regarding the contribution of DNA replication to ecDNA formation. One report showed that during replication, ecDNA can originate from loop excision and/or ligation of DNA fragments in the replication bubble where DNA replication is paused [32]. In contrast, other studies showed that inhibitors of DNA replication promoted the formation of ecDNA [33]. In addition, the DNA fragments released into peripheral blood by damaged cells in response to oxidative stress further contribute to ecDNA formation [34-36]. Collectively, the formation of ecDNA involves complex mechanisms (Fig. 1).

\section{Source sequences}

Studies have shown the source sequences of ecDNA originate from multiple genomic sites from various individual chromosomes [23]. Storlazzi et al. demonstrated that ecDNA exhibits a high degree of heterogeneity in the sequence source, even within a single cell [37, 38]. Bioinformatic analyses of ecDNA sequences also indicate that oncogene amplification is unlikely to be the consequence of chromothripsis [23] (Fig. 2).

\section{The oncogenic functions of ecDNA}

Recent studies have demonstrated fundamental roles of ecDNA in cancer in modulating cell growth $[15,19,39]$, metastasis/invasion [40, 41], autophagy [42, 43], DNA damage repair [34, 35], drug response [40,44] and clinical outcome [15, 41] (Fig. 3). In addition, ecDNA contributes to intra-tumoral heterogeneity through genetic, epigenetic and microenvironmental factors $[1,2,13,18]$.

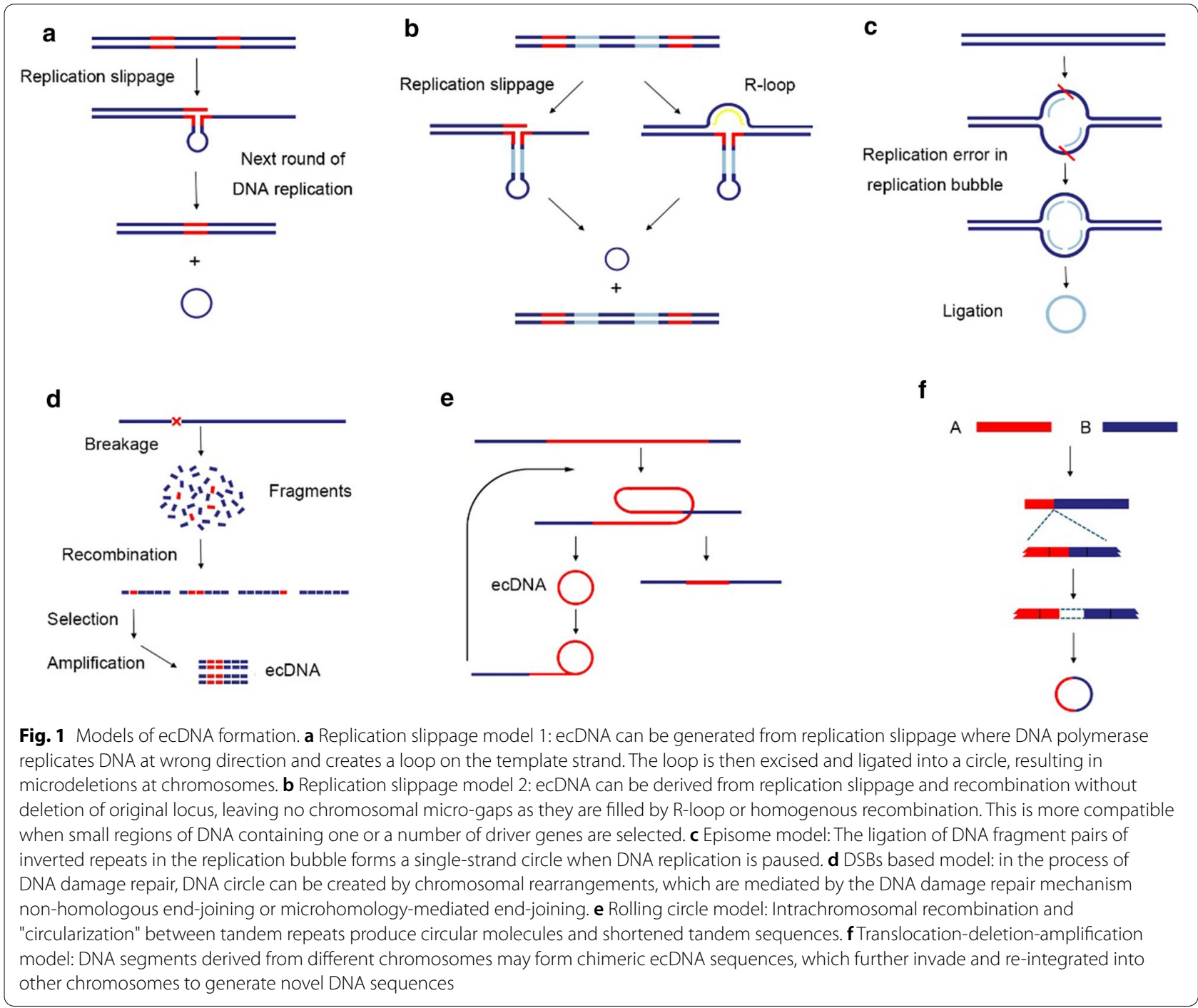




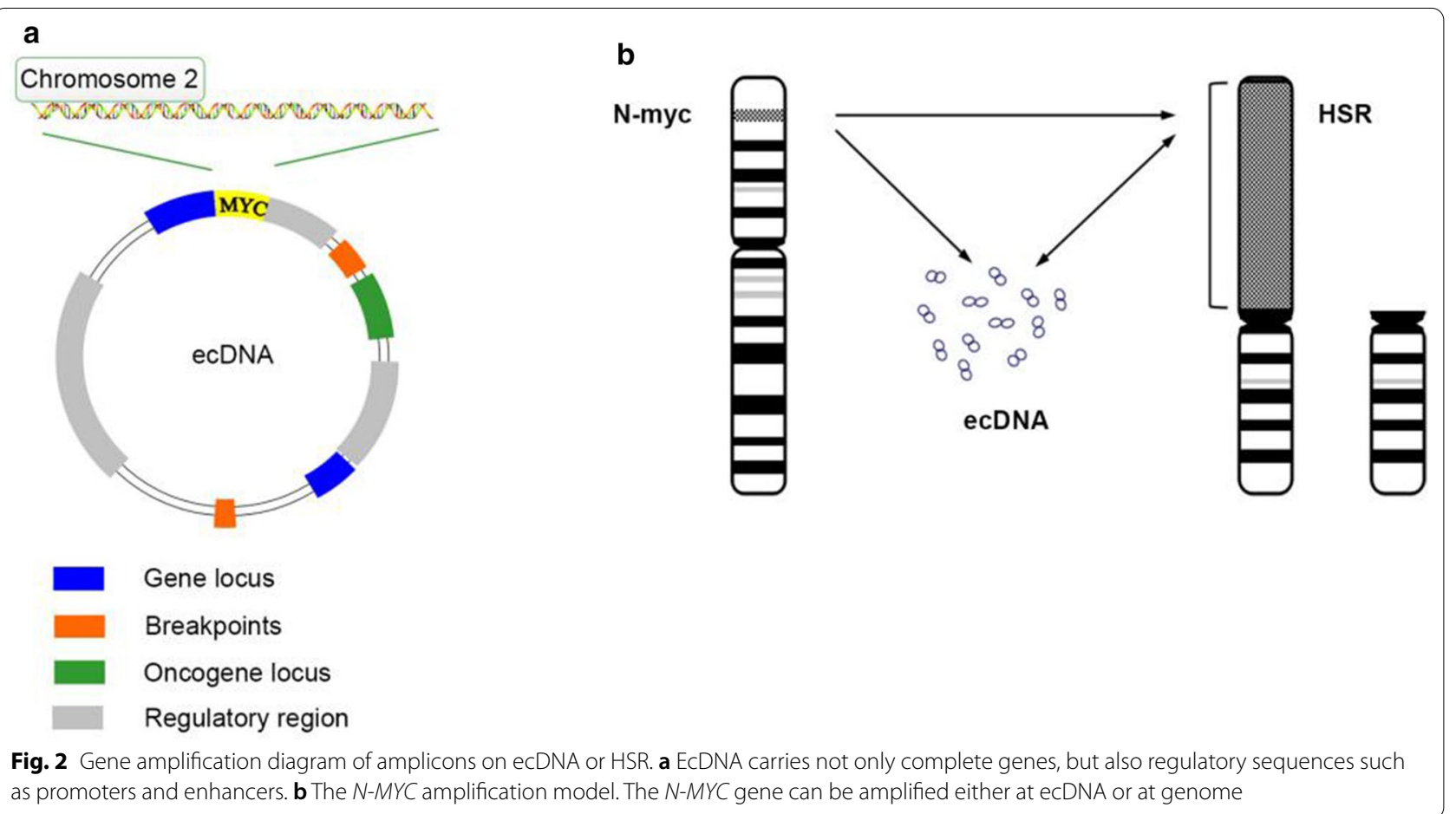

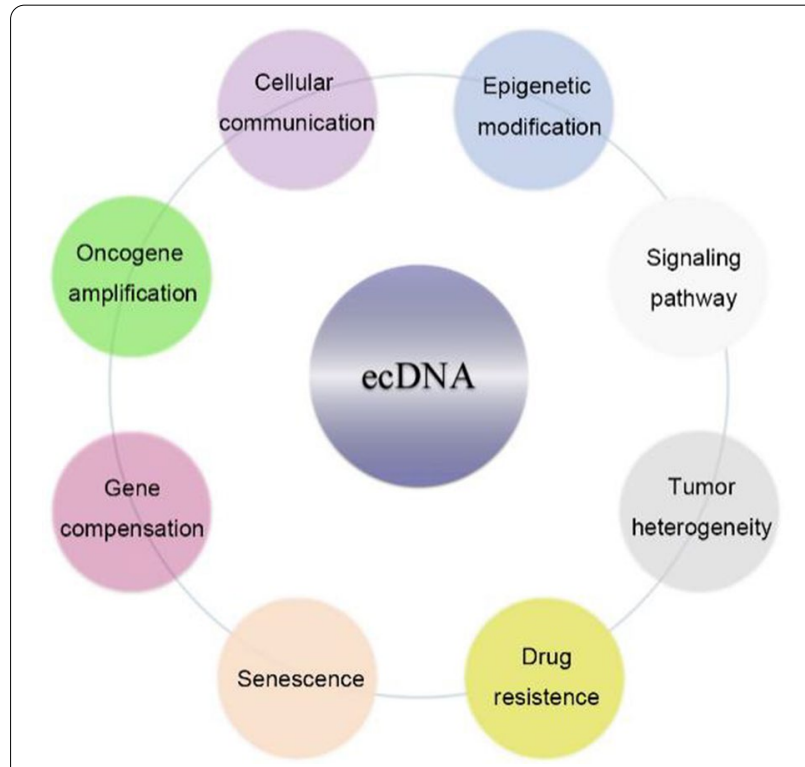

Fig. 3 Overview of the oncogenic functions of ecDNA. EcDNA has a profound impact on multiple aspects of cancer phenotypes

\section{Cell growth}

The formation of ecDNA correlates with enhanced levels of DNA replication in highly proliferating cancer cells and exhibits survival benefits [2, 41]. In addition, ecDNA enhances the proliferation of cancer cells but suppresses the infiltration of immune cells, thus leading to an aggressive phenotype of elevated number of lymph nodes with micro-metastases in cancer patients [15].

\section{Intra-tumoral heterogeneity}

Several studies showed that ecDNA increases the level of intra-tumoral heterogeneity in multiple cancer types $[1$, $2,12]$. The ecDNA originating from asymmetric chromatin segregation during mitosis and the massive amplification of oncogenes in ecDNA enable cancer cells to readily adapt to the evolving environment. Both primary and recurrent tumors show amplification of ecDNA-encoded genes (e.g. MYC, MYCN, EGFR, PDGFRA and MET), linking ecDNA to the evolvability of cancer cells under the selection pressure of the tumor microenvironment and therapeutic treatment [14]. In addition, as ecDNA is much more abundant in progressive tumors whereas high levels of HSR are more frequently observed in tumors under environmental stress, the balance between ecDNA and HSR of chromosomes is considered essential to determine the evolvability of cancer cells $[18,45]$.

\section{Autophagy}

Several reports showed that ecDNA activates pathogen recognition receptors such as Toll-like receptor (TLR) family proteins, leading to inhibition of apoptosis 
and promotion of autophagy $[42,44]$. In line with these results, some studies showed that ecDNA-containing cell-free DNA may regulate autophagy in a TLR9dependent manner [42, 44]. Furthermore, studies in colon cancer cells have demonstrated that ecDNA transported in micronuclei or extracellular vesicles (EVs) can facilitate the induction of autophagy thus to promote cancer cell survival in response to chemotherapy [44].

\section{Drug sensitivity}

Schimke et al. discovered that methotrexate resistance was attributed to DHFR gene amplification in ecDNA [22]. Meng et al. found that knock-down of DHFR resulted in increased sensitivity to methotrexate in DNAPKcs-depleted ecDNA-containing cells but not in HSRcontaining cells [46]. Glioblastoma cells have high levels of oncogenic EGFRvIII in ecDNA [18, 45]. Turner et al. performed structural analysis of EGFRvIII amplification on glioblastoma cells (GBM39) and found that ecDNA reintegrated into HSR during erlotinib treatment. Importantly, the ecDNA amplicon re-emerged when erlotinib was discontinued [18]. In addition, resistance to tyrosine kinase inhibitors of glioblastoma cells can be strengthened by adjustment of EGFRvIII levels in ecDNA [47]. These findings demonstrate that ecDNA modulates the drug sensitivity of cancer cells.

\section{Metastasis and invasiveness}

Recent findings have linked ecDNA to increased cancer metastasis and poor patient outcomes. The overall level of ecDNA is markedly elevated in cancer patients with metastases than in patients without metastases [40, 41]. Mechanistically, ecDNA shuttle between the nucleus and cytoplasm and can be encapsulated in micronuclei or transported in EVs to cross the cell membrane or be exported to the extracellular space by exosomes $[19,48]$. Cancer cells may use ecDNA as a messenger to transmit oncogenic information to other cell types in the microenvironment or to satellite tumors. ecDNA-mediated autocrine and paracrine signaling may result in increased invasiveness and chemoresistance and acquisition of the cancer stem cell-like phenotype [48]. In addition, ecDNA positively correlates with poor patient outcome [49]. The overall survival of patients carrying at least one circular amplicon of ecDNA was significantly poorer than that of patients without ecDNA-associated amplicons [15]. A meta-analysis of ecDNA measurement from 1076 patients with metastatic colorectal cancer confirmed a positive correlation between lower basal levels of ecDNA and better patient survival [41]. Furthermore, ecDNA can be used as a non-invasive prognostic tool that predicts the early relapse of thyroid cancer [50] and chemotherapy response in ovarian cancer [51].

\section{Senescence}

Senescence is a potent barrier to prevent the malignant transformation of normal cells to cancer cells [52]. EcDNA functions as a reservoir of heterogeneous genetic material that endows cancer cells with rapid adaptation to environmental stress [53]. In yeast, the induction of senescence can be attributed to the accumulation of ecDNA with ribosome genes [54]. Importantly, daughter cells with less ecDNA exhibited a longer lifespan than mother cells with more ecDNA, and the ectopic expression of autonomously replicating sequence of ecDNA can trigger cell cycle arrest, cell death or age-related inflammation [54, 55]. However, the underlying mechanisms of how ecDNA circumvents the barrier of senescence to facilitate malignant transformation remain to be elucidated.

\section{Anti-tumor immunity}

The elimination of ecDNA involves the entrapment of ecDNA within micronuclei, disappeared chromosomal $\gamma \mathrm{H} 2 \mathrm{AX}$ foci and the stimulation of immune responses [56]. Shimizu et al. found that ecDNA originating from anaphase chromosomes form micronuclei after hydroxyurea treatment [56]. Micronuclei facilitate the generation of aneuploid cells, which exhibit enhanced cell viability [57]. In neuroblastoma, ecDNA-containing micronuclei with amplified $M Y C N$ sequences were detected in vivo [58]. Notably, the DNA within micronuclei is prone to be released into the cytosol [59]. Dynamic for extracellular DNA interacts with micronuclei may be important for induction of anti-tumor immune response. Ji et al. found that downregulation of ecDNA-carried genes from colorectal and neuroectodermal tumor cells led to reduction of ecDNA genes by micronuclei expulsion which resulted in a decrease of tumor proliferation and malignancy [60]. As micronuclei are potential biomarkers for inflammation and DNA damage and known to trigger innate immune response including activation of cGAS-STING innate immune signaling [58], the cross-link of ecDNA and anti-tumor immunity is worth further investigation.

\section{The mechanistic actions of ecDNA Oncogene amplification}

Oncogene amplification is one of the driving factors of tumorigenesis and can occur at either the HSR structures on chromosomes or ecDNA [61]. Studies have reported significantly elevated copy numbers of oncogenes encoded in ecDNA (e.g. EGFR, $M Y C, C D K 4$, and MDM2) [2]. The amplification of oncogenes in ecDNA markedly increases overall oncogene expression, which can be found in both primary and metastatic tumors regardless of treatment [18]. In addition to elevating oncogene levels by copy number amplification, ecDNA may re-integrate 
into HSRs of chromosome and/or affect DNA accessibility to further "stabilize" the expression of oncogenes (e.g. EGFR in glioblastoma) [47].

The distinct inheritance pattern of ecDNA differs from the traditional Mendel's law of inheritance and raises the question of whether and how the location of amplified oncogenes impacts tumorigenesis. In this regard, Lobachev et al. found that the breaking sites of yeast chromosomes determine the consequences of gene amplification [62]. EcDNA is often observed to be produced from oncogene amplification, if the breaking sites locate between the hairpin break and the telomere. In contrast, when the break occurs between the oncogene and telomere, the amplification of oncogenes will generate HSR [62].

Importantly, a positive feedback regulatory loop between the elevated expression of ecDNA-encoded genes and the accumulation of ecDNA has emerged. Hull et al. found that yeast cells obtain high levels of ecDNA containing the copper resistance gene CUP1 under copper exposure, and CUP1 expression may cause further accumulation of CUP1-bearing ecDNA [63]. Moreover, Ji et al. showed that down-regulation of genes in ecDNA may result in the integration of ecDNA into cytoplasmic micronuclei and the subsequent reduction of ecDNA [60]. These results reveal a mechanistic link between the accumulation of ecDNA and oncogene hyper-activity.

\section{Chromosome rearrangements}

As one of the major sources of somatic rearrangements, ecDNA exemplifies the mutagenic feature of the cancer genome [64]. Chromosomal rearrangements include translocations and/or insertions, which often result in oncogenes adjoining to transcriptional regulatory elements (e.g. promoters, enhancers) and the formation of fusion genes [65-67].

\section{Rewiring enhancers}

Morton et al. found that enhancers of EGFR, including endogenous enhancers as well as rewired enhancers from topological-associated domains, were co-amplified with oncogenes in glioblastoma [3]. These selectively skewed enhancers were also found in multiple cancer types (e.g. medulloblastoma, neuroblastoma and Wilms tumors) [3]. Helmsauer et al. further demonstrated that the majority of genomic rearrangement events involved ecDNA, challenging the current understanding of cancer genome remodeling [64].

\section{Gene fusions}

Gene fusions in ecDNA have been widely observed in leukemia and solid tumors, such as multiple myeloma [67], medulloblastoma [66] and gastric cancer [65].
Graux et al. identified a novel mechanism for the activation of tyrosine kinases in which the formation of ecDNA resulted in gene fusion between NUP214 and ABL1 in T-cell acute lymphoblastic leukemia [68]. Additionally, amplification of the $B C R-A B L 1$ fusion gene on ecDNA and the translocation of $(9 ; 22)$ (q34;q11) have been reported in chronic myeloid leukemia during imatinib treatment $[69,70]$. Furthermore, L'Abbate et al. identified the PVT1 gene on ecDNA as a hotspot for breakpoints whose amplification and rearrangements positively correlated with drug resistance and poor patient outcome in small cell lung cancer, indicating a crucial role of ecDNA in gene fusions [23].

\section{Epigenetic modifications}

Epigenetic modifications, which includes the chemical modification of chromatin, gene compensation, chromatin interaction, and topological reconstruction, alter the accessibility of chromatin and ecDNA and play a key role in a variety of biological processes [2, 71, 72].

\section{Histone modification}

Previous studies showed that ecDNA are enriched with active rather than repressive histone markers [2]. Analyses of metaphase glioblastoma cells further demonstrated the high levels of active histone marks (H3K4me1, $\mathrm{H} 3 \mathrm{~K} 27 \mathrm{ac}$ ) on ecDNA, while the levels of repressive markers (H3K9me3, H3K27me3) were low [2].

\section{Gene compensation}

EcDNA plays a critical role in the compensation of histone genes. In Saccharomyces cerevisiae, a novel circular ecDNA with HTA2-HTB2 amplification was generated to compensate for the effects of HTA1-HTB1 deletion through the recombination between two Ty1 retrotransposon elements [72]. This finding suggests that loss of histone genes somehow activates a gene compensatory mechanism on ecDNA to maintain the proper expression levels of histone genes that are required for transcriptional activities.

\section{Nucleosome accessibility}

Topological studies have shown that ecDNA is packaged into circular chromatin and nucleosome units and lacks the canonical high-order of chromatin structure that is commonly seen in chromosomal DNA [2]. This unique structure of ecDNA leads to enhanced chromatin accessibility to transcriptional machineries to ecDNA-encoded genes [2].

\section{Remote chromatin interaction}

The circular chromosome conformation capture technology combining high-throughput sequencing (4C-seq) has 
been used to assess the chromatin connection on ecDNA [73]. Previous studies have shown that the remote interaction of active chromatin was enhanced via ecDNA, and even ultra-remote chromatin contact could be detected [2].

\section{Signaling pathways regulated by ecDNA}

Better understanding of signaling pathways regulated by ecDNA is essential to elucidate the biological functions of ecDNA in cancer. These signals influence oxidative stress, inflammation and the bystander effect (Fig. 4) [34, $35,74-76]$.

\section{Oxidative stress signaling and bystander effect}

ecDNA signaling contributes to the development of adaptive responses and bystander effect under oxidative stress. Low dose ionizing radiation triggers oxidative stress, DNA modification, apoptosis, ecDNA generation and subsequent changes in bystander cells [35]. The damaged DNA in irradiated cells can be released into the intracellular space and received by bystander cells through caspase 3 and TLR (e.g. TLR9) dependent mechanisms $[36,74,76]$. Consistent with the responses of irradiated cells to oxidative stress, bystander cells also show alterations in nuclei shape, activation of nucleolar organizer regions, promotion of actin polymerization and elevation of double-strand break level (i.e. bystander effect) [76]. Accordingly, the increased level of ecDNA stimulates the rapid synthesis of reactive oxygen and nitrogen species, resulting in secondary oxidative stress and upregulation of anti-oxidant genes (e.g. NRF2, KEAP1, SOD1) [75].

\section{Pro-inflammatory signaling}

The presence of ecDNA stimulates the production of pro-inflammatory cytokines that are deleterious to cancer cells [34]. Notably, ecDNA was shown to activate TLR9-MyD88-NF-kB signaling in the plasma of rheumatoid arthritis patients, leading to increases in pro-inflammatory cytokines (e.g. IL-6, TNF- $\alpha$ ) [77]. In addition, the high GC content of ecDNA appears to affect the production of pro-inflammatory cytokines. One study reported that the GC-rich elements of ecDNA, but not genomic DNA, activated the production of IL- 6 and TNF- $\alpha$ [77].

\section{Metabolic reprogramming signaling}

High EGFR expression was shown to drive glycolysis through EGFR signaling, PI3K pathway and $c-M Y C$ dysregulation [78]. EGFRvIII signaling is stringently regulated in the metabolic events of glioblastoma. EGFRvIII-dependent metabolic reprogramming includes the synergistic regulation of fatty acid synthesis through Akt-SREBP1-dependent mechanisms [79] and the control of intra-tumoral cholesterol levels through LDLRdependent signaling [80]. Importantly, $M Y C$ was shown to be co-amplified with $S Q L E$, a key metabolic gene that encodes squalene monooxygenase in the sterol biosynthesis pathway [81]. Furthermore, $M Y C$ also upregulates PYCRL, a crucial regulator of ornithine to proline conversion, and its isoenzymes to enhance the synthesis of proline [82].

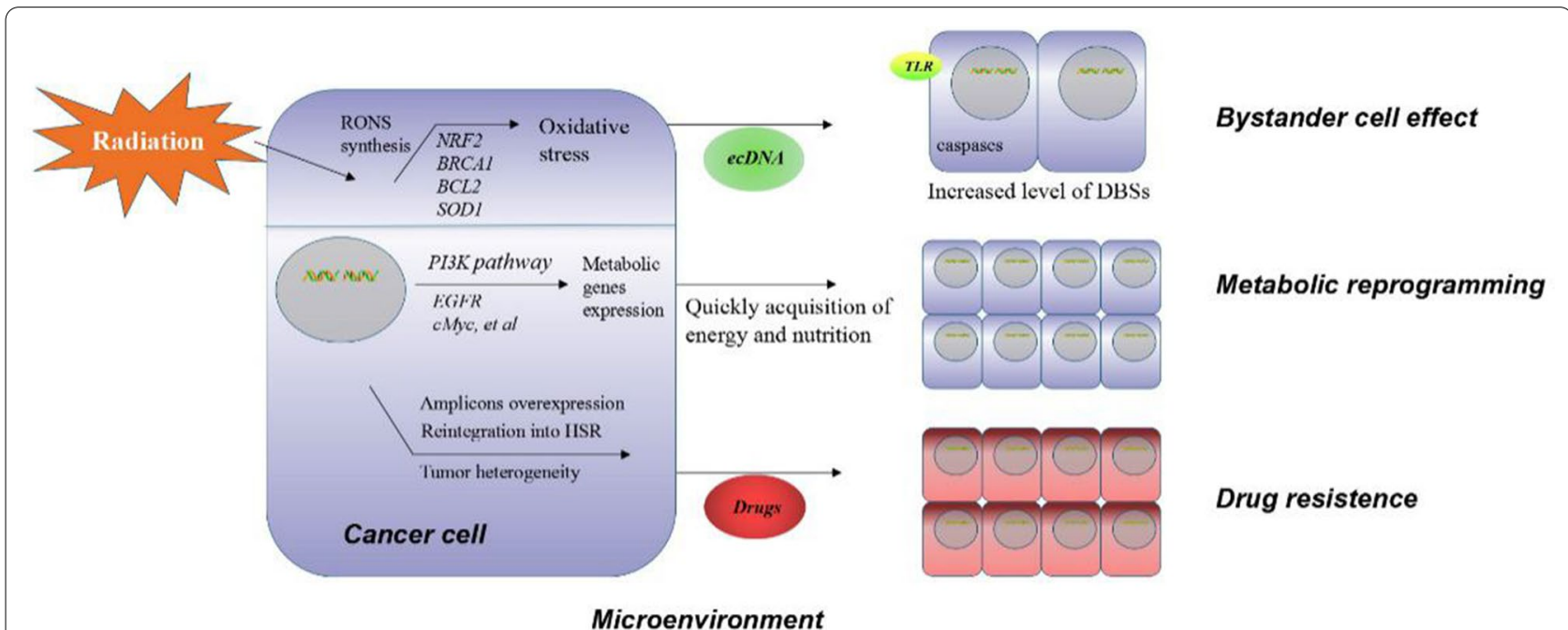

Fig. 4 Mechanisms of ecDNA signaling in cancer. In response to oxidative stress, ecDNA induces the activation of inflammation, and to exert the bystander effect. Also, ecDNA regulates the metabolic reprogramming signaling to enhance the acquisition of energy in cancer cells 


\section{ecDNA as a potential biomarker}

An analysis of over 3200 clinical samples revealed that ecDNA was found in at least $14 \%$ of human cancers [2]. The frequency of ecDNA is likely to be higher in most aggressive cancer types, including glioblastoma, neuroblastoma, hepatocellular carcinoma, leukemia, lung and ovarian cancer [83]. Recent studies have revealed the potential utility of ecDNA in tumor diagnosis, prognosis and potential treatment of certain cancers on clinic [84, 85] (Table 2). The relationship of oncogenes amplified on ecDNA with drug sensitivity is also summarized below.

ecDNA may represent a novel tool for various clinical applications mainly in three aspects. First, ecDNA can be released into the peripheral blood system [86] and may serve as potential prognostic biomarkers of multiple cancers, such as thyroid cancer [50], cervical cancer [87], ovarian cancer [88] and non-small cell lung cancer [89]. As an example, ecDNA has been used in liquid biopsy of thyroid cancer as a new plasma genotyping source [50]. Second, elimination of oncogenes on ecDNA increases drug sensitivity [22, 69, 90, 47], providing a novel adjunctive therapeutic option for chemotherapy. Third, ecDNA-carrying EVs transport oncogenes and trigger tumorigenesis [19]. Thus, detecting and targeting EVs might have potential utility for cancer treatment.

\section{Conclusions and perspectives}

Recent findings have revolutionized our understanding of ecDNA in cancer, highlighting the potential of ecDNA as a potential biomarker for personalized therapy. Since ecDNA is usually more stable than linear DNA, ecDNA may potentially be used in liquid biopsy [86]. However, the prognostic and/or diagnostic power of ecDNA remains undetermined. Clinical proof to support the feasibility of ecDNA as a biomarker is still lacking.

Table 2 The roles and potential applications of ecDNA in cancers

\begin{tabular}{|c|c|c|c|c|}
\hline Cancers & Current advances & \multicolumn{2}{|l|}{$\begin{array}{l}\text { The connection between ecDNA and clinical } \\
\text { applications }\end{array}$} & Refs. \\
\hline \multicolumn{5}{|c|}{ I. Serving as potential biomarker to assess clinical outcomes } \\
\hline Thyroid cancer & $\begin{array}{l}\text { Development of a noninvasive diagnostic tool for } \\
\text { biopsy }\end{array}$ & \multicolumn{2}{|l|}{$\begin{array}{l}\text { EcDNA is a component in liquid biopsy of thyroid } \\
\text { cancer as a new plasma genotyping source }\end{array}$} & {$[42]$} \\
\hline Cervical cancer & $\begin{array}{l}\text { Development of a computational diagnosis } \\
\text { method }\end{array}$ & \multicolumn{2}{|l|}{$\begin{array}{l}\text { The presence of ecDNA-viral structures is verified } \\
\text { in cervical cancer samples }\end{array}$} & {$[43]$} \\
\hline Ovarian cancer & Mouse xenograft model & \multicolumn{2}{|l|}{$\begin{array}{l}\text { Ciuculating DNA complements miRNAs and } \\
\text { linear DNA for diagnosis }\end{array}$} & {$[44]$} \\
\hline Non-small-cell lung cancer (NSCLC) & Application in the FLAURA phase III trial & \multicolumn{2}{|l|}{$\begin{array}{l}\text { Circulating tumor DNA (ctDNA) serves as primary } \\
\text { objective to depict genetic tumor profile }\end{array}$} & {$[45]$} \\
\hline Hepatocellular carcinoma (HCC) & $\begin{array}{l}\text { Study in biopsy and plasma samples in HCC } \\
\text { patients }\end{array}$ & \multicolumn{2}{|l|}{$\begin{array}{l}\text { ecDNA tracks real-time therapeutic responses } \\
\text { and could overcome tumor heterogeneity }\end{array}$} & {$[98]$} \\
\hline Cancers & Genes on ecDNA & Functions & Refs. & \\
\hline \multicolumn{5}{|c|}{ II. Elimination of oncogenes reside on ecDNA increases drug-sensitivity } \\
\hline Glioblastoma & MYC, EGFR, PDGFRa, ERBB2, CDK4, MDM2 & $\begin{array}{l}\text { Amplification of EGFRvIll results in erlotinib } \\
\text { resistance }\end{array}$ & \multicolumn{2}{|l|}{$[48]$} \\
\hline Colon cancer & $D H F R, C-M Y C$ & $\begin{array}{l}\text { Down-regulation of DHFR on ecDNA increases } \\
\text { MTX sensitivity }\end{array}$ & \multicolumn{2}{|c|}{$[57,94,95]$} \\
\hline Neuroblastoma & MYCN & $\begin{array}{l}\text { Elimination of MYCN on ecDNA increases HU } \\
\text { sensitivity }\end{array}$ & \multicolumn{2}{|l|}{$[67]$} \\
\hline Cervical cancer & DHFR & Amplification of DHFR promote MTX resistance & \multicolumn{2}{|l|}{$[96]$} \\
\hline Ovarian cancer & MYCN, EIF5AR, CA125 & $\begin{array}{l}\text { Decreased levels of ecDNA-form CA125 after HU } \\
\text { treatment }\end{array}$ & \multicolumn{2}{|l|}{$[97]$} \\
\hline Breast cancer & DHFR, HER2 & $\begin{array}{l}\text { Loss of HER2 residing on ecDNA has no effect } \\
\text { on trastuzamab therapy }\end{array}$ & \multicolumn{2}{|c|}{$[98,99]$} \\
\hline Leukemia & $c-M Y C$ & $\begin{array}{l}\text { Down-regulation of c-MYC promotes drug } \\
\text { sensitivity }\end{array}$ & \multicolumn{2}{|l|}{ [100] } \\
\hline Oral squamous cell carcinoma & MDR1 & Loss of MDR1 enhances HU sensitivity & \multicolumn{2}{|l|}{$[101]$} \\
\hline \multicolumn{5}{|c|}{ III. Extracellular vesicles carrying ecDNA transfer oncogenes and trigger tumorigenesis } \\
\hline Ovarian cancer & $\begin{array}{l}\text { Studies of EVs from cancer cells remain in the } \\
\text { laboratory stage }\end{array}$ & \multicolumn{3}{|l|}{$\begin{array}{l}\text { ecDNA can be encapsulated in EVs. EVs might } \\
\text { have applications on clinic for tumor diagno- } \\
\text { sis, prognosis or potential treatment }\end{array}$} \\
\hline
\end{tabular}


Despite the promising findings, several aspects of ecDNA remain to be elucidated. Studies have shown the possible origin and destination of ecDNA [91, 92]; however, the type of stress that initiates the generation of ecDNA and whether and how the ecDNA-encoded genes could be selectively induced under the evolving microenvironment remain unclear. ecDNA may influence bystander cells in response to oxidative stress, but whether the original ecDNA-producing cancer cells affect bystander cells to facilitate tumorigenesis and/or progression is still unknown.

In addition, the functions of ecDNA in multiple biological processes (e.g. cell development, aging, genomic instability, adaptive evolution, drug resistance, tumor development) also need to be further investigated. Elucidation of the underlying mechanisms of ecDNA may further shed light on cancer therapeutics.

\begin{abstract}
Abbreviations
HSR: Homogeneously staining region; BFB cycle: Breakage-fusion-bridge cycle: EGFR: Epidermal growth factor receptor; DHFR: Dihydrofolate reductase; LDLR: Low density lipoprotein receptors; SREBP1: Sterol regulatory element binding protein-1; PYCRL: Pyrroline-5-carboxylatereductase L; DSB: DNA double strand break; EV: Extracellular vesicle.
\end{abstract}

\section{Acknowledgements}

This work was supported, in whole or in part, by The Program for Professor of Special Appointment (Eastern Scholar) at Shanghai Institutions of Higher Learning (No. TP2018046 to J.S.), Shanghai Municipal Education CommissionTwo Hundred Talents (No. 20191817 to J.S.), and General Program of National Natural Science Foundation of China (No. 81972667 to J.S.).

\section{Authors' contributions}

YHW, RH and GPZ performed the literature review, wrote the review and helped with the revisions. JFS wrote and edited the manuscript. All authors read and approved the final manuscript.

\section{Availability of data and materials}

Not applicable.

\section{Declarations}

Ethics approval and consent to participate

Not applicable.

\section{Consent for publication}

Not applicable.

\section{Competing interests}

The authors declare that they have no competing interests.

\section{Author details}

'Department of Ophthalmology, Ninth People's Hospital, Shanghai JiaoTong University School of Medicine, Shanghai 200025, China. ${ }^{2}$ Shanghai Key Laboratory of Orbital Diseases and Ocular Oncology, Shanghai 200025, China.

Received: 17 December 2020 Accepted: 13 April 2021

Published online: 26 April 2021

\section{References}

1. Verhaak RGW, Bafna V, Mischel PS. Extrachromosomal oncogene amplification in tumour pathogenesis and evolution. Nat Rev Cancer. 2019;19(5):283-8.

2. Wu S, Turner KM, Nguyen N, Raviram R, Erb M, Santini J, et al. Circular ecDNA promotes accessible chromatin and high oncogene expression. Nature. 2019:575(7784):699-703.

3. Morton AR, Dogan-Artun N, Faber ZJ, MacLeod G, Bartels CF, Piazza MS, et al. Functional enhancers shape extrachromosomal oncogene amplifications. Cell. 2019;179(6):1330-41.

4. Balaban-Malenbaum G, Gilbert F. Double minute chromosomes and the homogeneously staining regions in chromosomes of a human neuroblastoma cell line. Science. 1977;198(4318):739-41.

5. Buoen LC, Brand KG. Double-minute chromosomes in plastic filminduced sarcomas in mice. Sci Nat-Heidelberg. 1968;55(3):135-6.

6. Pristyazhnyuk IE, Menzorov AG. Ring chromosomes: from formation to clinical potential. Protoplasma. 2018;255(2):439-49.

7. Garsed DW, Marshall OJ, Corbin VDA, Hsu A, Di Stefano L, Schröder $J$, et al. The architecture and evolution of cancer neochromosomes. Cancer Cell. 2014;26(5):653-67.

8. Macchia G, Severgnini M, Purgato S, Tolomeo D, Casciaro H, Cifola I, et al. The hidden genomic and transcriptomic plasticity of giant marker chromosomes in cancer. Genetics. 2018;208(3):951-61.

9. Papenfuss AT, Thomas DM. The life history of neochromosomes revealed. Mol Cell Oncol. 2015;2(4):e1000698.

10. Qiu GH. Protection of the genome and central protein-coding sequences by non-coding DNA against DNA damage from radiation. Mutat Res Rev Mutat Res. 2015;764:108-17.

11. Paulsen T, Shibata Y, Kumar P, Dillon L, Dutta A. Small extrachromosomal circular DNAs, microDNA, produce short regulatory RNAs that suppress gene expression independent of canonical promoters. Nucleic Acids Res. 2019;47(9):4586-96.

12. Koche RP, Rodriguez-Fos E, Helmsauer K, Burkert M, MacArthur IC, Maag $J$, et al. Extrachromosomal circular DNA drives oncogenic genome remodeling in neuroblastoma. Nat Genet. 2019;12:16.

13. Deshpande V, Luebeck J, Nguyen ND, Bakhtiari M, Turner KM, Schwab $\mathrm{R}$, et al. Exploring the landscape of focal amplifications in cancer using AmpliconArchitect. Nat Commun. 2019;10:15.

14. DeCarvalho AC, Kim H, Poisson LM, Winn ME, Mueller C, Cherba D, et al. Discordant inheritance of chromosomal and extrachromosomal DNA elements contributes to dynamic disease evolution in glioblastoma. Nat Genet. 2018;50(5):708-17.

15. Kim H, Nguyen N, Turner K, Wu S, Gujar AD, Luebeck J, et al. Extrachromosomal DNA is associated with oncogene amplification and poor outcome across multiple cancers. Nat Genet. 2020;8:17.

16. Rajkumar U, Turner K, Luebeck J, Deshpande V, Chandraker M, Mischel P, et al. EcSeg: semantic segmentation of metaphase images containing extrachromosomal DNA. iScience. 2019;21:428-35.

17. Brettmann EA, Oh IY, de Guzman SC. High-throughput identification of gene regulatory sequences using next-generation sequencing of circular chromosome conformation capture (4C-seq). J Vis Exp. 2018;140:58.

18. Turner KM, Deshpande V, Beyter D, Koga T, Rusert J, Lee C, et al. Extrachromosomal oncogene amplification drives tumour evolution and genetic heterogeneity. Nature. 2017;543(7643):122-5.

19. Tandon I, Pal R, Pal JK, Sharma NK. Extrachromosomal circular DNAs: an extra piece of evidence to depict tumor heterogeneity. Fut Sci OA 2019;5(6):O390.

20. Kohl NE, Kanda N, Schreck RR, Bruns G, Latt SA, Gilbert F, et al. Transposition and amplification of oncogene-related sequences in human neuroblastomas. Cell. 1983;35(2):359-67.

21. Alitalo K, Schwab M, Lin CC, Varmus HE, Bishop JM. Homogeneously staining chromosomal regions contain amplified copies of an abundantly expressed cellular oncogene (c-myc) in malignant neuroendocrine cells from a human colon carcinoma. Proc Natl Acad Sci USA. 1983;80(6):1707-11.

22. Haber DA, Schimke RT. Unstable amplification of an altered dihydrofolate reductase gene associated with double-minute chromosomes. Cell. 1981;26(3):355-62.

23. L'Abbate A, Macchia G, D'Addabbo P, Lonoce A, Tolomeo D, Trombetta $D$, et al. Genomic organization and evolution of double minutes/ 
homogeneously staining regions with MYC amplification in human cancer. Nucleic Acids Res. 2014;42(14):9131-45.

24. Smith G, Taylor-Kashton C, Dushnicky L, Symons S, Wright J, Mai S. c-Myc-induced extrachromosomal elements carry active chromatin. Neoplasia. 2003;5(2):110-20.

25. MCCLINTOCK B. Chromosome organization and genic expression. Cold Spring Harb Symp Quant Biol. 1951;16:13-47.

26. Barr FG, Nauta LE, Davis RJ, Schafer BW, Nycum LM, Biegel JA. In vivo amplification of the PAX3-FKHR and PAX7-FKHR fusion genes in alveolar rhabdomyosarcoma. Hum Mol Genet. 1996;5(1):15-21.

27. Carroll SM, Gaudray P, De Rose ML, Emery JF, Meinkoth JL, Nakkim E, et al. Characterization of an episome produced in hamster cells that amplify a transfected CAD gene at high frequency: functional evidence for a mammalian replication origin. Mol Cell Biol. 1987;7(5):1740-50.

28. Stephens PJ, Greenman CD, Fu B, Yang F, Bignell GR, Mudie LJ, et al. Massive genomic rearrangement acquired in a single catastrophic event during cancer development. Cell. 2011;144(1):27-40.

29. Menghi F, Barthel FP, Yadav V, Tang M, Ji B, Tang Z, et al. The tandem duplicator phenotype is a prevalent genome-wide cancer configuration driven by distinct gene mutations. Cancer Cell. 2018;34(2):197-210.

30. Gisselsson D, Jin Y, Lindgren D, Persson J, Gisselsson L, Hanks S, et al. Generation of trisomies in cancer cells by multipolar mitosis and incomplete cytokinesis. Proc Natl Acad Sci USA. 2010;107(47):20489-93.

31. Korbel JO, Campbell PJ. Criteria for inference of chromothripsis in cancer genomes. Cell. 2013;152(6):1226-36.

32. Vogt N, Lefevre SH, Apiou F, Dutrillaux AM, Cor A, Leuraud P, et al. Molecular structure of double-minute chromosomes bearing amplified copies of the epidermal growth factor receptor gene in gliomas. Proc Natl Acad Sci USA. 2004:101(31):11368-73.

33. Paulsen T, Kumar P, Koseoglu MM, Dutta A. Discoveries of extrachromosomal circles of DNA in normal and tumor cells. TRENDS GENET. 2018;34(4):270-8.

34. Ermakov AV, Konkova MS, Kostyuk SV, Izevskaya VL, Baranova A, Veiko NN. Oxidized extracellular DNA as a stress signal in human cells. Oxid Med Cell Longev. 2013;2013:1-12.

35. Ermakov AV, Kon'Kova MS, Kostiuk SV, Veiko NN. DNA-signaling pathway mediating development of a radiation-induced bystander effect in human cells. Radiats Biol Radioecol. 2011;51(6):651-9.

36. Ermakov AV, Konkova MS, Kostyuk SV, Egolina NA, Efremova LV, Veiko NN. Oxidative stress as a significant factor for development of an adaptive response in irradiated and nonirradiated human lymphocytes after inducing the bystander effect by low-dose X-radiation. Mutat Res. 2009;669(1-2):155-61.

37. Storlazzi CT, Lonoce A, Guastadisegni MC, Trombetta D, D'Addabbo P, Daniele G, et al. Gene amplification as double minutes or homogeneously staining regions in solid tumors: origin and structure. Genome Res. 2010;20(9):1198-206.

38. Storlazzi CT, Fioretos T, Surace C, Lonoce A, Mastrorilli A, Strombeck B, et al. MYC-containing double minutes in hematologic malignancies: evidence in favor of the episome model and exclusion of MYC as the target gene. Hum Mol Genet. 2006;15(6):933-42.

39. Cohen Z, Bacharach E, Lavi S. Mouse major satellite DNA is prone to eccDNA formation via DNA Ligase IV-dependent pathway. Oncogene. 2006;25(33):4515-24.

40. Xu K, Ding L, Chang T, Shao Y, Chiang J, Mulder $\mathrm{H}$, et al. Structure and evolution of double minutes in diagnosis and relapse brain tumors. Acta Neuropathol. 2019;137(1):123-37.

41. Spindler KLG, Boysen AK, Pallisgård N, Johansen JS, Tabernero J, Sørensen MM, et al. Cell-free DNA in metastatic colorectal cancer: a systematic review and meta-analysis. Oncologist. 2017;22(9):1049-55.

42. Múzes G, Kiss AL, Tulassay Z, Sipos F. Cell-free DNA-induced alteration of autophagy response and TLR9-signaling: their relation to amelioration of DSS-colitis. Comp Immunol Microbiol Infect Dis. 2017;52:48-57.

43. Liu J, Zhu Z, Liu Y, Wei L, Li B, Mao F, et al. MDM2 inhibition-mediated autophagy contributes to the pro-apoptotic effect of berberine in $\mathrm{p53-}$ null leukemic cells. Life Sci. 2020;242:117228.

44. Anunobi R, Boone BA, Cheh N, Tang D, Kang R, Loux T, et al. Extracellular DNA promotes colorectal tumor cell survival after cytotoxic chemotherapy. J Surg Res. 2018;226:181-91.

45. Tuveson D, Clevers H. Cancer modeling meets human organoid technology. Science. 2019;364(6444):952-5.
46. Meng X, Qi X, Guo H, Cai M, Li C, Zhu J, et al. Novel role for non-homologous end joining in the formation of double minutes in methotrexateresistant colon cancer cells. J Med Genet. 2015;52(2):135-44.

47. Nathanson DA, Gini B, Mottahedeh J, Visnyei K, Koga T, Gomez G, et al. Targeted therapy resistance mediated by dynamic regulation of extrachromosomal mutant EGFR DNA. Science. 2014;343(6166):72-6.

48. Sadovska L, Santos CB, Kalnina Z, Line A. Biodistribution, uptake and effects caused by cancer-derived extracellular vesicles. J Circ Biomark. 2015;4:2.

49. Bailey C, Shoura MJ, Mischel PS, Swanton C. Extrachromosomal DNA - relieving heredity constraints, accelerating tumour evolution. Ann Oncol. 2020;4:7.

50. Khatami F, Tavangar SM. Liquid biopsy in thyroid cancer: new insight. Int J Hematol Oncol Stem Cell Res. 2018;12(3):235-48.

51. Kalavska K, Minarik T, Vlkova B, Manasova D, Kubickova M, Jurik A, et al. Prognostic value of various subtypes of extracellular DNA in ovarian cancer patients. J Ovarian Res. 2018;11(1):85.

52. Calcinotto A, Kohli J, Zagato E, Pellegrini L, Demaria M, Alimonti A. Cellular senescence: aging, cancer, and injury. Physiol Rev. 2019;99(2):1047-78

53. Hull RM, Houseley J. The adaptive potential of circular DNA accumulation in ageing cells. Curr Genet. 2020:4:15.

54. Sinclair DA, Guarente L. Extrachromosomal rDNA circles-a cause of aging in yeast. Cell. 1997;91(7):1033-42.

55. Storci G, Bacalini MG, Bonifazi F, Garagnani P, De Carolis S, Salvioli S, et al. Ribosomal DNA instability: an evolutionary conserved fuel for inflammaging. Ageing Res Rev. 2020;58:101018.

56. Shimizu N, Misaka N, Utani K. Nonselective DNA damage induced by a replication inhibitor results in the selective elimination of extrachromosomal double minutes from human cancer cells. Genes Chromosom Cancer. 2007:46(10):865-74.

57. Mansilla S, Bataller M, Portugal J. A nuclear budding mechanism in transiently arrested cells generates drug-sensitive and drug-resistant cells. Biochem Pharmacol. 2009;78(2):123-32.

58. Valent A, Benard J, Clausse B, Barrois M, Valteau-Couanet D, TerrierLacombe $\mathrm{MJ}$, et al. In vivo elimination of acentric double minutes containing amplified MYCN from neuroblastoma tumor cells through the formation of micronuclei. Am J Pathol. 2001;158(5):1579-84.

59. Kwon J, Bakhoum SF. The cytosolic DNA-sensing CGAS-STING pathway in cancer. Cancer Discov. 2020;10(1):26-39.

60. Ji W, Bian Z, Yu Y, Yuan C, Liu Y, Yu L, et al. Expulsion of micronuclei containing amplified genes contributes to a decrease in double minute chromosomes from malignant tumor cells. Int I Cancer. 2014;134(6):1279-88.

61. Schimke RT. Gene amplification in cultured animal cells. Cell. 1984;37(3):705-13.

62. Lobachev KS, Rattray A, Narayanan V. Hairpin- and cruciform-mediated chromosome breakage: causes and consequences in eukaryotic cells. Front Biosci. 2007;12:4208-20.

63. Hull RM, King M, Pizza G, Krueger F, Vergara X, Houseley J. Transcriptioninduced formation of extrachromosomal DNA during yeast ageing. Plos Biol. 2019;17(12):e3000471.

64. Koche RP, Rodriguez-Fos E, Helmsauer K, Burkert M, MacArthur IC, Maag $J$, et al. Extrachromosomal circular DNA drives oncogenic genome remodeling in neuroblastoma. Nat Genet. 2020;52(1):29-34.

65. Kim HP, Cho GA, Han SW, Shin JY, Jeong EG, Song SH, et al. Novel fusion transcripts in human gastric cancer revealed by transcriptome analysis. Oncogene. 2014;33(47):5434-41.

66. Northcott PA, Shih DJ, Peacock J, Garzia L, Morrissy AS, Zichner T, et al. Subgroup-specific structural variation across 1000 medulloblastoma genomes. Nature. 2012;488(7409):49-56.

67. Nagoshi H, Taki T, Hanamura I, Nitta M, Otsuki T, Nishida K, et al, Frequent PVT1 rearrangement and novel chimeric genes PVT1-NBEA and PVT1-WWOX occur in multiple myeloma with 8q24 abnormality. Cancer Res. 2012;72(19):4954-62.

68. Graux C, Cools J, Melotte C, Quentmeier H, Ferrando A, Levine R, et al. Fusion of NUP214 to ABL1 on amplified episomes in T-cell acute lymphoblastic leukemia. Nat Genet. 2004;36(10):1084-9.

69. Barnes DJ, Palaiologou D, Panousopoulou E, Schultheis B, Yong $A S$, Wong $A$, et al. Bcr-Abl expression levels determine the rate of 
development of resistance to imatinib mesylate in chronic myeloid leukemia. Cancer Res. 2005;65(19):8912-9.

70. Morel F, Bris MJ, Herry A, Calvez GL, Marion V, Abgrall JF, et al. Double minutes containing amplified bcr-abl fusion gene in a case of chronic myeloid leukemia treated by imatinib. Eur J Haematol. 2003;70(4):235-9.

71. Corces MR, Granja JM, Shams S, Louie BH, Seoane JA, Zhou W, et al. The chromatin accessibility landscape of primary human cancers. Science. 2018:362(6413):v1898.

72. Libuda DE, Winston F. Amplification of histone genes by circular chromosome formation in Saccharomyces cerevisiae. Nature. 2006;443(7114):1003-7.

73. Zhao Z, Tavoosidana G, Sjölinder M, Göndör A, Mariano P, Wang S, et al. Circular chromosome conformation capture (4C) uncovers extensive networks of epigenetically regulated intra- and interchromosomal interactions. Nat Genet. 2006;38(11):1341-7.

74. Kostyuk SV, Ermakov AV, Alekseeva AY, Smirnova TD, Glebova KV, Efremova $L V$, et al. Role of extracellular DNA oxidative modification in radiation induced bystander effects in human endotheliocytes. Mutat Res. 2012;729(1-2):52-60.

75. Loseva P, Kostyuk S, Malinovskaya E, Clement N, Dechesne CA, Dani $C$, et al. Extracellular DNA oxidation stimulates activation of NRF2 and reduces the production of ROS in human mesenchymal stem cells. Expert Opin Biol Ther. 2012;12:S85-97.

76. Ermakov AV, Konkova MS, Kostyuk SV, Smirnova TD, Malinovskaya EM, Efremova LV, et al. An extracellular DNA mediated bystander effect produced from low dose irradiated endothelial cells. Mutat Res. 2011;712(1-2):1-10.

77. Speranskii Al, Kostyuk SV, Kalashnikova EA, Veiko NN. Enrichment of extracellular DNA from the cultivation medium of human peripheral blood mononuclears with genomic $\mathrm{CpG}$ rich fragments results in increased cell production of IL-6 and TNF-a via activation of the NF-kB signaling pathway. Biomed Khim. 2016;62(3):331-40.

78. Bi J, Wu S, Zhang W, Mischel PS. Targeting cancer's metabolic codependencies: A landscape shaped by genotype and tissue context. Biochimica et Biophysica Acta. 2018;1870(1):76-87.

79. Guo D, Prins RM, Dang J, Kuga D, Iwanami A, Soto H, et al. EGFR signaling through an Akt-SREBP-1-dependent, rapamycin-resistant pathway sensitizes glioblastomas to antilipogenic therapy. Sci Signal. 2009;2(101):a82.

80. Guo D, Reinitz F, Youssef M, Hong C, Nathanson D, Akhavan D, et al. An LXR agonist promotes glioblastoma cell death through inhibition of an EGFR/AKT/SREBP-1/LDLR-dependent pathway. Cancer Discov. 2011;1(5):442-56.

81. Haider S, McIntyre A, van Stiphout RG, Winchester LM, Wigfield S, Harris AL, et al. Genomic alterations underlie a pan-cancer metabolic shift associated with tumour hypoxia. Genome Biol. 2016;17(1):140.

82. Liu W, Hancock CN, Fischer JW, Harman M, Phang JM. Proline biosynthesis augments tumor cell growth and aerobic glycolysis: involvement of pyridine nucleotides. Sci Rep. 2015;5:17206.

83. Wu S, Bafna V, Mischel PS. Extrachromosomal DNA (ecDNA) in cancer pathogenesis. Curr Opin Genet Dev. 2021;66:78-82.

84. Yan Y, Guo G, Huang J, Gao M, Zhu Q, Zeng S, et al. Current understanding of extrachromosomal circular DNA in cancer pathogenesis and therapeutic resistance. J Hematol Oncol. 2020;13:12.

85. Liao Z, Jiang W, Ye L, Li T, Yu X, Liu L. Classification of extrachromosomal circular DNA with a focus on the role of extrachromosomal DNA (ecDNA) in tumor heterogeneity and progression. Biochimica et Biophysica Acta. 2020;1874(1):188392.

86. Khatami F, Larijani B, Tavangar SM. The presence of tumor extrachomosomal circular DNA (ecDNA) as a component of liquid biopsy in blood. Med Hypotheses. 2018;114:5-7.

87. Nguyen ND, Deshpande V, Luebeck J, Mischel PS, Bafna V. ViFi: accurate detection of viral integration and mRNA fusion reveals indiscriminate and unregulated transcription in proximal genomic regions in cervical cancer. Nucleic Acids Res. 2018;46(7):3309-25.

88. Kumar P, Dillon LW, Shibata Y, Jazaeri AA, Jones DR, Dutta A. Normal and cancerous tissues release extrachromosomal circular DNA (eccDNA) into the circulation. Mol Cancer Res. 2017;15(9):1197-205.

89. Bennouna J, Girard N, Audigier-Valette C, le Thuaut A, Gervais R, Masson $\mathrm{P}$, et al. Phase II study evaluating the mechanisms of resistance on tumor tissue and liquid biopsy in patients with EGFR-mutated non-pretreated advanced lung cancer receiving osimertinib until and beyond radiologic progression: The MELROSE Trial. Clin Lung Cancer. 2020;21(1):e10-4.

90. Morales C, Ribas M, Aiza G, Peinado MA. Genetic determinants of methotrexate responsiveness and resistance in colon cancer cells. Oncogene. 2005;24(45):6842-7.

91. Gu X, Yu J, Chai P, Ge S, Fan X. Novel insights into extrachromosomal DNA: redefining the onco-drivers of tumor progression. J Exp Clin Cancer RES. 2020;39:18.

92. Wei J, Wu C, Meng H, Li M, Niu W, Zhan Y, et al. The biogenesis and roles of extrachromosomal oncogene involved in carcinogenesis and evolution. Am J Cancer Res. 2020;10(11):3532-50.

93. Cai ZX, Chen G, Zeng YY, Dong XQ, Lin MJ, Huang XH, et al. Circulating tumor DNA profiling reveals clonal evolution and real-time disease progression in advanced hepatocellular carcinoma. Int J Cancer. [Journal Article; Research Support, Non-U.S. Gov't]. 2017 ;141(5):977-85.

94. Cai M, Zhang H, Hou L, Gao W, Song Y, Cui X, et al. Inhibiting homologous recombination decreases extrachromosomal amplification but has no effect on intrachromosomal amplification in methotrexateresistant colon cancer cells. Int J Cancer. [Journal Article; Research Support, Non-U.S. Gov't]. 2019;144(5):1037-48.

95. Shimizu N, Itoh N, Utiyama H, Wahl GM. Selective entrapment of extrachromosomally amplified DNA by nuclear budding and micronucleation during S phase. J Cell Biol. [Journal Article; Research Support, Non-U.S. Gov't; Research Support, U.S. Gov't, Non-P.H.S.]. 1998;140(6):1307-20.

96. Ruiz-Herrera A, Smirnova A, Khoriauli L, Nergadze SG, Mondello C, Giulotto E. Gene amplification in human cells knocked down for RAD54. Genome Integr. [Journal Article]. 2011;2(1):5.

97. Zhang CY, Feng YX, Yu Y, Sun WJ, Bai J, Chen F, et al. The molecular mechanism of resistance to methotrexate in mouse methotrexateresistant cells by cancer drug resistance and metabolism Super Array. Basic Clin Pharmacol Toxicol. [Journal Article; Research Support, NonU.S. Gov't]. 2006;99(2):141-5.

98. Hahn P, Nevaldine B, Morgan WF. X-ray induction of methotrexate resistance due to dhfr gene amplification. Somat Cell Mol Genet. [Journal Article; Research Support, U.S. Gov't, Non-P.H.S.; Research Support, U.S. Gov't, P.H.S.]. 1990;16(5):413-23.

99. Vicario R, Peg V, Morancho B, Zacarias-Fluck M, Zhang J, MartinezBarriocanal A, et al. Patterns of HER2 gene amplification and response to Anti-HER2 therapies. Plos One. [Journal Article; Research Support, Non-U.S. Gov't]. 2015;10(6):e129876.

100. Eckhardt SG, Dai A, Davidson KK, Forseth BJ, Wahl GM, Von Hoff DD. Induction of differentiation in HL60 cells by the reduction of extrachromosomally amplified c-myc. Proc Natl Acad Sci USA. [Journal Article; Research Support, Non-U.S. Gov't; Research Support, U.S. Gov't, P.H.S.]. 1994;91(14):6674-8.

101. Von Hoff DD, Waddelow T, Forseth B, Davidson K, Scott J, Wahl G. Hydroxyurea accelerates loss of extrachromosomally amplified genes from tumor cells. Cancer Res. [Journal Article; Research Support, NonU.S. Gov't; Research Support, U.S. Gov't, P.H.S.]. 1991;51(23 Pt 1):6273-9.

\section{Publisher's Note}

Springer Nature remains neutral with regard to jurisdictional claims in published maps and institutional affiliations. 